\title{
Permeation of Rule Awareness Education in Physical Education of Higher Vocational Colleges
}

\author{
Shun Li Gao \\ Weifang Medical University, China \\ Email: gaoshunli6723@126.com
}

Keywords: Rule Awareness; Physical Education; Vocational colleges; Sports Games.

\begin{abstract}
It is one of important tasks for higher education to train socialism qualified builders and reliable successors with rule awareness. Rules consciousness is even more important in vocational education of higher vocational college particularly due to specialty of educational objects thereof. Physical education, as an important part of vocational education, is mutually integrated with rule awareness, which is more conducive to development of vocational education and beneficial for training vocational students.
\end{abstract}

\section{Introduction}

Construction of China's socialist market economic system and law society is inseparable from rule awareness. Any groups, as large as a country or a re-gion and as small as a unit, a class or a family, should require members thereof to abide by given code of conduct or guidelines in order to maintain normal order and promote own healthy development. There is a saying in China that nothing can be accomplished without norms or standards. Kant once said: 'Human beings love order, so the human beings create rules for the world'. College stage acts as an important period for forming a person's outlook on world, outlook on life and value, which is also important for educators to firmly control talent training and rule awareness training. Physical Education, as an important part in higher vocational college education, plays an important role in higher vocational education; integration of rule awareness into physical education is objective requirement of physical education and development direction in the future.

\section{Concept of awareness education}

Meaning of Rule Awareness. Rule is code of basic regulation and requirement for guiding people to engage in relevant behaviors in society, maintaining social order, and providing corresponding relief for victims. Rule awareness refers to awareness refers to the awareness from the heart with rule as own behavior criterion. It is reflection of social existence, social individuals or citizens will produce different rule ideologies at different social development stages and under different social structures. Citizen rule awareness is an important aspect of citizen awareness connotation.

Importance of Rule Awareness. Rule awareness can not be formed automatically, which should depend on acquired education and training. School education is an important part in many educational pathways. The Moral Education Course naturally becomes main channel of rule awareness education since it is closest to requirement of rule awareness education in the aspects of teaching contents and teaching objective. Rule awareness education has become a common blind spot in our ideological and moral education. Therefore it is urgent to conduct rule awareness education in vocational school education at present due to the subsequence of the education blind spot.

It is undeniable that vocational school student level is significantly declined in recent years from the perspective of vocational school student source characteristics, most students fail in general education examination. There is certain proportion of students with weak cultural foundation and behavior habits. Their study motivation is complex and diverse, and students are lack of self-confidence generally with low judgment recognition ability on surrounding matters. They are 
extremely impulsive in viewing problems with poor self-control ability. Some students suffer from distorted value orientation, are slack and lack of discipline, repeatedly offend school disciplines and school rules, or even conduct crimes such as robbery extortion and gang fights. It is obvious that rule awareness deficiency is extremely prominent in the crowd.

Vocational school graduates are just eighteen years old from the perspective of vocational school student age characteristics. They not only have to complete the learning tasks, but also should convert from minors to adults and from students to social roles within three years. Children during the age always feel confused, dazed and disoriented during transition from more relaxed school environment with simple interpersonal relationship to adult world with fierce competition and complicated interpersonal relationship. Compliance with the group rule is the easiest and most effective adaptation mode, thereby obtaining group recognition and acceptance.

Most vocational school students will engage in front-line operation job due to limitation of academic level from the perspective of vocational school student employment pressure. Enterprise pays more attention to spirit of dedication rather than knowledge level thereof in employment standard aiming at frontline workers since quality of frontline workers directly affect customer satisfaction for enterprise. Dedication, observation of labor discipline, implementation of regulated workflow and quality standards can become key factors for vocational school students to win in employment market with fierce competition. The above analysis clearly shows that it is urgent and very important to carry out rules awareness education in vocational schools indeed. It is related to vocational stability and future development of vocational school students, and status of vocational education in the educational system of the entire community. Rule awareness education in schools is comprehensive, in-cluding efforts in the aspects of moral teaching, spe-cialized course teaching, class management, practice activities, campus culture building, etc. Moral course teaching is the main channel of rule awareness education in various approaches. We should make good use of the main channel for cultivating rule awareness of vocational school students.

\section{Role of rule awareness in college physical education}

College students experience the stage of rapid physical and psychological change, and are highly adaptable. Rule awareness refers to a series of long-term work; anticipated education goal can not be completely simply by implementation of some education activity.

Practicality is the unique attribute of sport disci-pline teaching. PE teaching is mainly a practical cur-riculum that motion technology is regarded as the core content physical exercise is regarded as the main means, rule restriction competition is regarded as organization form, physical and mental forces are combined together under different time periods, space and natural conditions in dynamic state, image and abstract thinking are highly fit, interpersonal relationship is extremely broad and active. Therefore, physical education provides rule education with occasion, chances, living textbook, scenario, role playing, etc., which can not be replaced by other disciplines in the school. Rule education permeation in physical education is not empty and armchair strategy, which replaces slogan with practice on the basis of student behavior practice. The philosophy concept of 'easier said than done' is reflected. Practicability of Physical Education itself is a complex, changing and dynamic system. Teachers should organize rigorously and scientifically with certain rule requirement, which plays excellent situational education role for training student rule awareness.

Another major feature with more prominent performance in physical education is sociability. Sociability in physical education always refers to interaction or mutual activity between teachers and student, and among students. There is close link between teachers and student and among students in physical education rather than closed acceptance of education knowledge for single student. It is the wide exchange and mutual action between teachers and students, and among students that trains non-intelligence factors of students, thereby creating practice platform for rule education. Physical education modes are generally divided into collective exercise, group exercise, etc. Sports competition forms include competition among individuals, competition between individual and the 
collective, intercollegiate competition, etc. Exchange among students in physical education in the aspects of time, space and scenario can not be replaced by other disciplines. Exchange provides the fundamental legitimate foundation for rules. Rule awareness can be obtained through physical education.

Regularity is main feature of physical education content. School physical education teaching contents still keeps essence of item competition rules after certain textbook-based modification in competition sports items and leisure items. Otherwise origin connotation of sports items is lost. Contest rule is soul reflection of sports All sports all have their own strict rules, competition participants must strictly adhere to these rule details, for example students can receive 0 score even the record is broken due to pedal rule violation in standing long-jump. The tiny error of surface phenomenon on the surface can lead to serious result. After students enter into the society, the tiny rule restriction is found everywhere. Students can conduct a rehearsal for future employment through rule education in physical competition. Therefore, teachers should consciously guide students to understand and follow specific rules in sports activities in physical education process. Students violating the rule should be punished, and students creating high achievement by following rules should be awarded. Therefore, students can comprehend and stimulate social contract spirit and civilized lifestyle through physical education. However, students can not form ethical rules and obtain social adaptability by simply comprehending and imitating competition rules in physical education activities. Students can integrate the simulation in individual behavior, namely, forcible behavior rules can be subtly integrated into own behavior habits and act criteria. Rule consciousness formed during physical education pro-cess is beneficial for early socialization process of students.

\section{Approach of higher vocational colleges to implement physical education}

Sports classroom discipline is the basic approach for physical education to implement rule education. Main place of physical education is classroom. Good classroom discipline is necessary to ensure smooth teaching process. Sports classroom discipline is means and behavior that teachers and students jointly obey classroom behavior rules in physical education, physical education teachers should maintain normal teaching education, students are encouraged to participate in physical education activities actively, and illegal behaviors should be organized and punished. Discipline and rule are twin brothers, rule acts as recognition on rule and behavior orientation of rule. Nothing is available without discipline during physical education teaching. Teachers require students to exercise ulti-mately aiming at training students into socialist suc-cessors through physical education teaching and other discipline education jointly, and the students should love the motherland and the collective, follow discipline, and be honest, brave, wise and progressive. Gathering, queuing, adjustment and other requirements in physical education teaching rules require consistency and coordination, and neat steps of students, which is beneficial for training students' habits to follow discipline. Some items in Physical Education are strongly competitive, confrontational and unpredictable, etc. If students fail to comply with classroom discipline in the process of teaching, harm incidents can be produced, such as standing posture in shot teaching, protection in gymnastics teaching, etc. In addition, students must wear sneakers in physical education class. Students who do not wear the sneakers should be responsible for own behaviors. These classroom disciplines are certainly not sudden or random so as to ensure the effectiveness and seriousness of the rules. Therefore, students can experience difficulty in physical education activity without discipline, and 'violation' should be punished.

Sports game is entertainment approach for physical education to implement rule education. It is important content in physical education, mainly including activities promoting general development of body, special games closely related with sports specialized technology, and most activities are in entertainment competition forms. Destroy of game situations and game order is not allowed in sports game activities, game can not be implemented smoothly without game situation and order. Rules are necessary for game aiming at game, and game players must follow rules in order to participate in 
game. Sport game rules and other details regulate game activity style. It also focuses that game players must follow behavior rules in time and space as restriction. For example, penalty rule adds entertaining atmosphere of game on the one hand, it also strengthens behavior rule of game participants on the other hand. Game punishment is the climax of sports game activities, teachers should guide students to recognize and understand the purpose and significance of game penalty correctly. It is clear that the game penalty not only acts as one form of teaching activity, but also performs as entertainment climax of physical education game activity. Sports game also has individual socialization enlightenment function; therefore the effect can be durable by regarding sports game permeated to student mind as means for rule education. Teachers can allow students to conduct sufficient and bold creative activity in rule scope during rule education of sports game. Sports game contents and rules can be modified and perfected constantly according to game concrete condition in the implementation process, thereby improving game interest and achieving the education purpose of making students accept rule awareness education in game. Sport game permeation is utilized for rules education on students. Students' behavior habit of transacting according to rule can be trained at meantime. They can establish the awareness of consciously abiding by various rules. Importance of rule in human activities and restriction of the society on human activities are made clear. It is essential education step for each student who will enter the society soon.

Sports competition is an important approach for physical education to implement rule education. The rule is microcosm of the society on member norm and ethical conducts, and important part of physical education. The core value of contemporary sports produces huge influence on human development in society, economy, politics and other aspects, human emotion and values through competition-the focused social behavior. Sports competition is conducted in scope limited by various separate, concrete and strict sports competition rules. Clear rules can be applied in the competition process for unifying standards, adjusting scale, and regulating behavior. Competition rule refers to law or regulation for implementing any one specific sports competition smoothly. Competition rule provides one equal opportunity for every participant. It allows participant to devote wholehearted effort for a meaningful effort, thereby completing freely selected task. Rule rigorousness is just prominent feature of competitive sports; random change of rule must destroy unique charm of competition. Compliance with competition rules aims at better reflecting reasonable, intense and funny features of competitive sports contests, and making competitive sports items more viable. Physical education restricts student behavior through sports competition rules, thereby subtly enhancing culture of social ethics regulation. Competition rules have the nature of simulating social regulation. Each student should follow rule in specific competition time and space, thereby forming recognized value subtly. The abiding value is demanded in our era, which has important realistic significance to promote the building of socialist harmonious society in China.

\section{Summary}

Establishment of harmonious society is inseparable from rule awareness. Good wishes of law society are established on human respect on rule and love on or-der. Surely, training of rule awareness can not be completed overnight. It should exert classroom disci-pline, sports game and student language rule and be-havior norm in sports competition during physical education. The contents are visible and tangible, integration and mutual permeation of object and sub-ject is beneficial for integrating the contents into stu-dent rule awareness, thereby making school rule actually become scale and yardstick of college student learning and life.

\section{References}

[1] Peng Wenjun, Yang Chunhua. Reform and Development Stratege on P.E.in Ethical Dilemmas[J].Journal of Longdong University.2013,24(5):86-88. 
[2] LI Ying. Progress on PE Teaching Ethics Study[J]. Journal of Tianjin Agricultural University. 2011,18(4):57-61.

[3] Yi Jiandong. Logical bases and value orientation of sport system reform in China[J]. Journal of Physical Education. 2011,(1):15-18.

[4] Kang Jianglong. Tactics to Implement the Physical and Mental Quality Education in Vocational Physical Education[J]. Journal of Ningbo Instituture of Education.2013,15(4):91-94.

[5] Yang Xiaoming. On New Thinking about the Harmonious Society and PE Education in the Countryside[J]. Journal of Lianyungang Teachers College.2007,2:78-80.

[6] Xu Longcheng. On the Coupling between PE Education and Moral Education[J]. Journal of Gannan Teacher's College.2004,3:45-47. 